> La communauté scientifique internationale a attendu plus de trente-cinq ans pour reconnaître la valeur du travail théorique et expérimental de Gregor Mendel (1822-1884). Nous proposons une nouvelle interprétation des faits et des documents ayant déterminé son devenir entre 1847 et 1849, qui permet de suggérer que sa personnalité était bien différente de celle décrite par ses biographes. <

\section{Une maladie énigmatique dans la vie de Gregor Mendel}

\section{Christiane Nivet}

\section{L'œuvre et la personne de Gregor Mendel}

L'article de Gregor Mendel (1866) intitulé « Expériences sur les hybrides de plantes » frappe non seulement par la clarté de la conception et la qualité du travail réalisé, mais aussi par l'élégance et l'intelligence de sa présentation [1]. Cette forte impression est bien traduite par les propos de C. Stern [2] tenus à l'occasion du centenaire de cette publication: «Ce court traité est un des triomphes de l'esprit humain. II n'annonce pas seulement la découverte de faits importants au moyen de nouvelles méthodes d'observation et d'expérimentation. Mais, dans un geste de haute créativité, il décrit ces faits dans un cadre conceptuel qui leur donne un sens général. L'article de Mendel n'est pas seulement un document historique, il est encore d'une totale actualité en tant que suprême exemple de procédure expérimentale scientifique et de profonde intelligence des résultats ». Le désir de connaître l'homme, sa vie et la façon dont il a mené son œuvre mène à la lecture de sa biographie.

Deux biographies de Gregor Mendel font autorité, toutes deux écrites par des scientifiques d'origine morave. La plus récente, publiée par V. Orel [3], a été jugée par P.J. Bowler [4] digne de devenir l'unique source d'informations sur la vie et la carrière de Mendel, remplaçant ainsi la biographie, autrefois favorite, mais d'après lui dépassée, publiée par H. Iltis [5]. En réalité, comme nous allons le montrer, les deux ouvrages sont aussi utiles l'un que l'autre pour connaître la vie de Mendel.

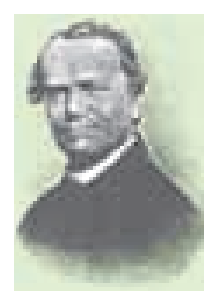

Contrastant avec les éloges adressés à son œuvre, le portrait de Gregor Mendel qui se dégage de ces ouvrages est surprenant: le personnage décrit a un caractère incertain, coutumier de désordres psychologiques qui auraient été à l'origine de son échec en tant que curé de paroisse. Ces troubles sont utilisés pour expliquer la nature de ses maladies d'enfance et peut-être aussi, vers la fin de sa vie, ses démêlés avec le pouvoir, après qu'il eut rejeté une taxation, jugée par lui abusive, des biens du monastère. Ce portrait d'une personnalité à la limite de la cohérence paraît peu compatible avec les importantes responsabilités dont il a été chargé ultérieurement, ni avec la ténacité et la constance exigées par la mise en œuvre de l'expérimentation entreprise, de façon indiscutée, sous sa seule responsabilité et pendant de longues années. On s'attend, au contraire, à rencontrer un personnage à l'ordonnance mentale impeccable.

L'origine de ces assertions sur la personnalité de Mendel pourrait être une lettre écrite par son supérieur pour informer l'évêque local de la mutation du prêtre de paroisse Mendel à un poste professoral en 1849. L'importance de cette lettre vient du fait que la vie de Gregor Mendel est difficile à connaître car on ne dispose pas de ses papiers personnels, brûlés peu après son décès. Les documents que les biographes ont pu utiliser sont donc essentiellement d'origine officielle: ils proviennent en grande partie des archives du monastère Saint-Thomas de Brünn en Moravie (actuellement Brno, 
République tchèque) où Mendel a vécu à partir de 1843 ; d'autres documents proviennent des administrations gouvernementales provinciales et impériales, la Moravie étant alors province d'Empire des Habsbourg. Dans ces conditions, l'interprétation des documents prend une importance capitale dans l'établissement des faits et de leur signification; elle joue un rôle plus grand encore dans la détermination des intentions et de l'état d'esprit de ceux qui y ont participé. Nous allons voir un exemple de la façon dont on peut interpréter la lettre mentionnée plus haut: afin d'en comprendre le sens, il faut au préalable rappeler les événements qui ont marqué la vie de Mendel au cours des années 1847 à 1849.

\section{Une période clé dans la vie de Mendel}

Dans l'impossibilité matérielle d'entreprendre des études supérieures, Mendel est entré en 1843 au monastère Augustin Saint-Thomas à Brünn. II reconnaît dans son autobiographie [6] qu'il espérait ainsi poursuivre ses études universitaires à la charge de cette institution. Le supérieur de Saint-Thomas, l'abbé C.F. Napp, qui cherchait de son côté des candidats professeurs capables d'enseigner les découvertes scientifiques les plus récentes, l'avait sélectionné en raison de ses qualités intellectuelles et de son intérêt pour l'étude des sciences. Avant d'être envoyé à l'université pour y acquérir la formation d'enseignant, Mendel accepte de suivre quatre années d'études théologiques afin de devenir prêtre.

Toutefois, à la fin de ses études de théologie, au lieu d'aller à l'université, Mendel est nommé contre son gré curé de la paroisse d'Altbrünn. En effet, en 1847, les paroissiens d'Altbrünn et les malades de l'hôpital voisin sont privés brutalement de curé, or le service de cette paroisse incombe aux prêtres du monastère Saint-Thomas, tous engagés dans de lourdes charges d'enseignement. Bien qu'en troisième année d'études de théologie, Mendel accède à la prêtrise et peut ainsi contribuer immédiatement au service de la paroisse.

Au cours du mouvement insurrectionnel du printemps 1848, plusieurs moines de Saint-Thomas, amis de Gregor Mendel, participent activement au soulèvement. Ils s'opposent ainsi ouvertement à l'évêque local, le comte Schaffgotsch, favorable au maintien du pouvoir établi. L'un des moines, le père Matous Klacel, professeur de philosophie et brillant polémiste politique, rédige une véhémente pétition réclamant pour les moines le droit d'orienter librement leur carrière ecclésiastique soit vers le ministère pastoral, soit vers l'enseignement et l'étude des sciences [7]. Mendel signe cette pétition publique, où il affirme sa volonté de changer d'affectation, en ajoutant après son nom la mention « candidat professeur ».

À l'automne 1848, l'insurrection est écrasée. Une forte réaction politique s'amorce tandis que Mendel reste curé de paroisse. II tombe très gravement malade en janvier 1849. Sans autre information, on apprend qu'à la rentrée des classes à l'au- tomne suivant, Mendel est nommé professeur suppléant dans une autre ville.

\section{Une lettre déconcertante}

V. Orel présente cette nomination comme résultant de l'entremise de Napp et mentionne l'existence d'une lettre de l'abbé, datée du 4 octobre 1849, informant l'évêque que le Présidium provincial a nommé Gregor Mendel professeur. V. Orel cite ensuite la fin de la lettre où l'abbé dit de Mendel qu'il est un «prêtre collégial [qui] mène une vie très retirée, modeste, vertueuse et religieuse, tout à fait appropriée à sa condition, mais aussi [qu'il] est très doué dans l'étude des sciences; cependant, il a beaucoup moins d'aptitudes en tant que prêtre de paroisse, la raison en étant qu'il est saisi par une incoercible timidité quand il doit se rendre au chevet des malades ou simplement contempler quiconque étant malade ou souffrant. En fait, cette infirmité l'a rendu très gravement malade, et c'est pourquoi j'ai trouvé nécessaire de le relever du service paroissial ».

C'est en s'appuyant sur cette lettre que le biographe s'est permis de conclure que, en raison de troubles psychologiques innés, Gregor Mendel n'a pu assumer le ministère d'assistance spirituelle auprès de ses paroissiens souffrants. II est probable que la grave maladie dont a souffert Mendel en janvier 1849 est celle dont l'abbé Napp parle dans sa lettre, mais si V. Orel donne des informations sur sa gravité (il dut rester alité pendant plus d'un mois et être veillé nuit et jour par une infirmière) et mentionne l'autorisation spéciale de dépense qu'il a fallu obtenir pour en régler les frais, il ne dit rien de la nature de la maladie en question.

Si H. Iltis, l'autre biographe de Mendel, tire de la lettre de Napp d'octobre 1849 le même genre de conclusions défavorables quant au psychisme de Mendel, il cite pourtant des documents et des faits, absents du récit d'Orel, qui permettent de remettre en cause ces conclusions.

\section{Levée des incohérences}

Hugo Iltis, qui a pu rencontrer des contemporains de Mendel au début du siècle dernier et recueillir des témoignages directs, dit, en commentaire de la lettre de Napp, dont il donne aussi le texte, que l'attitude timorée de Mendel devant les malades ne correspond pas du tout à son caractère décrit par ailleurs.

Le biographe fait l'aveu qu'il est « impossible actuellement de connaître la nature de la maladie contractée par Mendel lors de son travail au chevet des malades et des mourants ». À nos yeux, cette phrase indique que, curé de paroisse et aumônier de l'hôpital voisin, Mendel aurait été contaminé au cours de cette activité. La nature des soins reçus, décrits par Orel, évoque elle aussi une maladie plus physique que mentale. Par ailleurs, $\mathrm{H}$. Iltis nous apprend que le déficit en prêtres, cause de la nomination inat- 
tendue de Mendel à la paroisse, était due au décès de trois d'entre eux cette année-là (1847). On peut ainsi facilement en déduire que ce travail pastoral exposait les moines à un risque de contamination, ce qui suggère fortement que la maladie de Mendel a probablement été de nature infectieuse.

Enfin, dans le texte de la lettre de Napp rapporté par Iltis, on découvre que l'instance (le Présidium) qui a nommé Gregor Mendel professeur, contrairement à ce que prétend Orel, n'est pas de rang provincial mais impérial: cette version des faits est confirmée par le texte de la lettre officielle qui nomme Mendel dans l'enseignement, que le biographe Orel n'a pas jugé bon de mentionner.

\section{La lettre authentique de nomination}

Cette lettre, datée du 28 septembre 1849, écrite et signée de la main même du ministre d'empire Lazansky, a un caractère impératif d'application immédiate. En voici le texte qui concerne Mendel: «Une septième classe va être ouverte à l'école supérieure de Znaïm pour laquelle la municipalité fournira les fonds nécessaires. En rapport avec ces changement, on aura besoin d'un professeur supplémentaire... En raison du zèle que vous avez montré, je pense qu'il est juste de vous nommer professeur adjoint dans cette école et je vous enjoins de vous rendre sur place immédiatement, afin de vous présenter aux enseignants qui s'y trouvent et d'y prendre votre travail ».

Tout conduit à penser que Mendel a assuré courageusement ses fonctions de curé à la paroisse d'Altbrünn et auprès des malades de l'hôpital. II a rendu service à sa communauté en assurant l'intérim et, en récompense, on lui attribue selon ses vœux un emploi professoral.

Cette lettre émanant non seulement d'une administration impériale, mais du ministre lui-même, comme l'atteste la signature holographe, personne ne semble pouvoir empêcher le jeune prêtre de se rendre à son poste. C'est pourquoi, quelques jours plus tard, Napp, au lieu de demander l'autorisation de l'évêque pour changer l'affectation de Mendel, n'a plus, comme il le dit dans le début de sa lettre non rapporté par Orel, que le devoir d'informer celui-ci de la décision du ministre impérial. On peut alors s'interroger sur le but qu'il poursuit lorsqu'il mentionne «l'incoercible timidité devant les malades », le sort de Mendel étant réglé et mené à réalisation immédiate par la lettre du ministre du 28 septembre.

\section{L’objectif réel de la lettre de Napp}

Mendel n'a encore reçu ni la formation universitaire, ni l'habilitation de l'ćtat pour devenir professeur titulaire. La lettre du ministre lui permet seulement de quitter son affectation paroissiale, mais ne garantit pas la stabilité dans le poste.

Dans le contexte local morave, une lutte ouverte existe entre l'abbé et l'évêque à la suite des événements insurrectionnels de 1848. Lutte qui se laisse entrevoir dans le ton du début, cité par Iltis, de la lettre envoyée par Napp à Schaffgotsch au sujet de Mendel: «Je vous informe, comme j’y suis obligé... », puis au milieu de sa lettre: «Je me contenterai d'ajouter... ». II a fallu tout le poids de la lettre du ministre pour permettre à Mendel de quitter le travail paroissial en outrepassant le pouvoir du comte Schaffgotsch. Celui-ci, frustré dans son autorité, gardera un profond ressentiment à l'égard de ce jeune moine rebelle, mais vulnérable car, contrairement aux autres moines signataires de la pétition et qui étaient des professeurs chevronnés, Mendel n'était encore qu'un prêtre sans qualification pour enseigner.

On peut suggérer que Napp, poussant l'avantage politique que lui donne l'intervention du ministre, tente de rendre irréversible la mutation de Mendel vers le professorat. II transforme donc la grave maladie contagieuse de Mendel en une maladie qui proviendrait d'une infirmité innée « qui l'a rendu gravement malade », de façon à faire réformer définitivement ce jeune curé encore inexpérimenté et dont on peut prétendre qu'il se révèle, à l'usage, incapable d'effectuer un des ministères essentiels de la fonction pastorale. L'inaptitude, une fois reconnue, devient irréversible. Cette hypothèse prend en compte l'absence de documents sur la nature de la maladie de Mendel qui, n'étayant pas le propos de Napp, ont probablement été supprimés sous sa responsabilité, laissant seulement à disposition celui ou ceux qui attestent de sa gravité, servant en cela le projet du prélat.

En fait, Mendel n'a aucune garantie de rester dans l'enseignement: l'intérêt d'un prêtre pour l'étude de la reproduction des êtres vivants, trop proche de la sexualité, est mal toléré par l'évêque. À la première occasion, il aura de fortes chances d'être renvoyé à la vie pastorale dans une campagne reculée, comme l'ont été, avant lui, les professeurs qui avaient tenté d'échapper à la censure impériale religieuse. L'abbé Napp, passionné par le problème de la transmission des caractères héréditaires à travers les générations, a vu en Mendel, dès cette époque, la personne capable d'en trouver la clé; avant tout, il fallait lui en assurer les moyens matériels.

\section{Conclusions : qui était Mendel ?}

Nous en sommes arrivés à supposer que la lettre de l'abbé Napp ne donne pas une description exacte des aptitudes pastorales réelles de Mendel, mais au contraire une version erronée pour dégager définitivement Mendel du statut de curé. D'après l'appréciation du ministre Lazansky, nous savons que Mendel a servi fidèlement ses paroissiens d'Altbrünn. II a donc été un prêtre de paroisse honorable.

La thèse présentée par les deux biographes incontournables, selon laquelle le psychisme de Mendel aurait été perturbé de façon innée, est fondée sur la lettre de Napp, dont on sait qu'il impute à une impuissance psychologique la grave maladie contractée par Mendel en janvier 1849. En réalité, Mendel a 
probablement été contaminé lors de son assistance aux malades de l'hôpital: Mendel aurait donc été un homme sain d'esprit pouvant correctement remp ir son office.

Dans le contexte de violente réaction politique qui a suivi la révolution de 1848, la lettre de Napp, responsable de la mauvaise réputation de Mendel en tant qu'homme et en tant que curé, est néanmoins le seul moyen trouvé par le supérieur pour donner à Mendel une situation matérielle lui permettant de mener à bien son expérimentation.

II y a déjà bien longtemps, dans une revue critique de la biographie écrite par IItis, W. Bateson [8] s'est opposé ouvertement aux thèses pour le moins discourtoises sur le psychisme de Gregor Mendel, refusant d'accepter, sans un sérieux complément d'information, les allégations relatives à cette inccercible timidité de Mendel devant les malades et à la grave maladie induite par une présumée hypocondrie. Malgré tout, la thèse des troubles psychiques de Mendel, acceptée par Iltis et entretenue par Orel, est restée une sorte de vérité officielle qui, bien qu'elle l'ait protégé efficacement de son vivant, a été à l'origine d'une réputation douteuse devant la postérité. Car les allégations des biographes ont été reprises par les historiens des sciences, plus intéressés par l'œuvre que par la personne, tels que F. Weiling [9] et R.C. Olby [10]. On les retrouve sous une forme moins hostile dans le seul livre sur Mendel en français publié par Orel et Armogathe [11].

La persistance de cette croyance inexacte ne peut s'expliquer que par la présence d'intérêts dont la nature est à préciser: il plane sur la vie de Gregor Mendel comme une sorte de malédiction qui se perpétue dans les temps présents. $\Delta$

\section{SUMMARY}

An enigmatic disease in Gregor Mendel's life

The great value of the experimental and theoretical work of Gregor Merdel has been recognized more than thirty five years after its publication; in this article, we suggest that his personality has still to be rediscovered. $\diamond$

\section{RéFÉRENCES}

1. Mendel G. Versuche über Pflanzen-Hybriden. In: Verhandlungen des Naturforschenden Vereines. Abhandlungen. Brünr 1866; 4: 3-47. Traduction anglaise dans Stern C, Sherwood ER, eds. The origin of genetics. A Mendel source book. San Francisco, CA: W. H. Freeman, 1966.

2. Stern C. Forewords. In: Stern C, Sherwood ER, eds. The origin of genetics. A Mendel source book. San Francisco, CA: W.H. Freeman, 1965.

3. Orel V. Gregor Mendel: the first geneticist. New Yorh: Oxforc University Press, 1995.

4. Bowler PJ. Putting the peas in context. Nature 1995; 378: 130.

5. Ilt s H. Gregor Johann Menciel: Leben, Werk und Wirkung. Springer: Berlin, 1924 Traduction anglaise, Life of Mendel. New Ycrk: Norton, 1932. $2^{\mathrm{e}}$ ed, New York: Hafner, 1956.

6. Mendel G. Autobiography, 1850. Traduction anglaise, IItis A. Gregor Mendel's autobiogrcphy. J Hered 1954; 45: 231-4.

7. Orel V, Verbik A. Mendel's involvement in the plea for freedom on teaching in the revolutionary year of 1848. Folia Mendeliana $1984 ; 19: 223-33$.

8. Bateson W. Mendeliana. Nature 1925; CXV: 481-6.

9. Weiling F. Historical study: Johann Gregor Mendel, 1822-1884. Am J Med Genet $1991 ; 40: 1-25$

10. Olby RC. Origins of Mendelism. London: Constable, 1966. $2^{\mathrm{e}}$ ed, Chicago: University of Chicago Press, 1985.

11. Orel V, Arrogathe IR. Menciel, un ir.connu célèbre. Paris: Belin, 1985.

\section{TIRÉS À PART}

C. Nivet

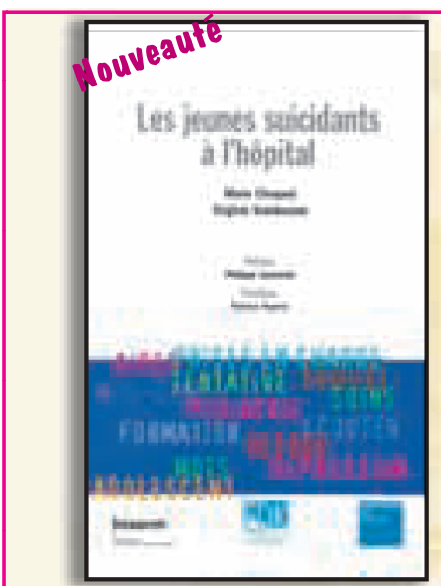

ISBN : 2-84254-093-X 204 pages e suicide constitue la deuxième cause de décès pour les 15-24 ans, et ce malg-é une diminution des taux de suicide d'environ $15 \%$ depuis 1985. Ainsi, actuellement, les jeunes meurent plus par suicide en France qu'en Italie ou en Grèce, aux Pays-Bas, au Portugal ou au Royaume-Uni. Quant à la morb dité suicidaire (tentative de suicide non suivie de décès), le problème persiste et s'aggrave même. Là encore, la France n'est pas en bonne position. Une enquête européenne a montré qu'entre 1989 et 1992 l'incidence a diminué en moyenne de $18 \%$ pour les garsons et de $11 \%$ pour les filles de 15 à 24 ans, sauf en France où elle a augmenté.

Face à ce constat, la Fondation de France a pris l'initiative de lancer l'enquête « Jeunes Suicidants à l'Hôpital » sous la direction de Marie Choquet, épidémiologiste, directeur de recherche à I'Inserm, et Virginie Granboulan, pédopsychiatre, praticien hospitalier au Centre hospitalier intercommunal de Créteil.

Un remarquable ouvrage qui permet de mieux connaître ces jeunes « en mal de vivre » et surtout de bousculer bien des idées reçues les concernant...

Préface de Philippe Jeammet

\begin{tabular}{|c|c|c|}
\hline \multirow{10}{*}{ 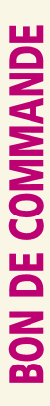 } & À retourner à EDK, 10 Villa d'Orléans - 75014 PARIS & Je souhaite recevoir l'ouvrage : \\
\hline & Têl. : 0153910606 - Fax : 0153910607 - E-mail : edk@edk.tr & Les jeunes suicidants à l'hôpital : \\
\hline & NOM : . & Prix public $18 €+3 €$ de port $=\mathbf{2 1} € \mathbf{T T C}$ \\
\hline & Adresse : ................. & \\
\hline & Code postal : ................................. Ville : & Par chèque, à l'ordre de EDK \\
\hline & Adresse e-mail : ............................... & $\square$ Eurocard/Mastercard \\
\hline & Tél. : ............. & Carte $n^{\circ} \quad \begin{array}{llllllllllllllllllll}1 & 1 & 1 & 1 & 1 & 1 & 1 & 1 & 1 & 1 & 1 & 1 & 1 & 1 & 1 & 1 & 1 & 1 & 1 & 1\end{array}$ \\
\hline & 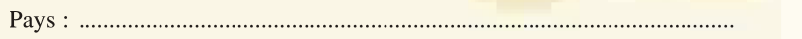 & \\
\hline & 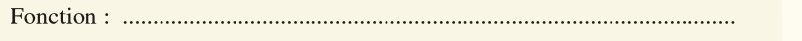 & Date d expiration : \\
\hline & & Signature : \\
\hline
\end{tabular}

